Artículo Original

\title{
Maternidad, migración y prematuridad: experiencias en una unidad de neonatología
}

\author{
Maternity, migration and prematurity: experiences in a neonatal care \\ unit
}

\section{Maternidade, migração, e prematuridade: experiências em uma unidade de neonatologia}

\author{
Andrea Mira ${ }^{\mathrm{a}}$ (D), Rodolfo Bastías ${ }^{\mathrm{b}}$ (D) \\ ${ }^{a}$ Universidad Andrés Bello - UNAB, Santiago, Chile. \\ ${ }^{\text {b} H o s p i t a l ~ L u i s ~ T i s n e ́, ~ S a n t i a g o, ~ C h i l e . ~}$
}

Cómo citar: Mira, A. \& Bastías, R. (2021). Maternidad, migración y prematuridad: experiencias en una unidad de neonatología. Cadernos Brasileiros de Terapia Ocupacional, 29, e2946.

https://doi.org/10.1590/2526-8910.ctoAO2232

\begin{abstract}
$\underline{\text { Resumen }}$
Los procesos migratorios son un fenómeno global que puede resultar en condiciones de vida más vulnerables. Dadas estas condiciones es que se hace relevante brindar los apoyos necesarios para las familias en situación de migración, donde algunos momentos críticos para esto serán el embarazo, el parto y la crianza. Es así como las mujeres migrantes viven su maternidad en un contexto cultural distinto y que dadas las condiciones de vida desfavorables pueden enfrentar dificultades en el embarazo, tales como el parto prematuro, lo que podría representar una experiencia traumática y estresante. Existen pocos estudios que aborden estas temáticas en Latinoamérica y es por esto que el objetivo de este estudio es describir las vivencias de madres migrantes con un hijo o hija de pretérmino hospitalizado/a en una unidad de neonatología. Se utilizó una metodología cualitativa con un enfoque fenomenológico. Se realizaron entrevistas en profundidad a 16 madres y de éstas emergieron temáticas relevantes tales como las diferencias culturales sobre la maternidad y crianza, las condiciones de vida, sus experiencias en el parto, y finalmente lo que significa ser madre es en un país diferente al suyo. Estos hallazgos permiten tener un mejor entendimiento sobre este fenómeno y podría facilitar el desarrollo de estrategias que favorecerían una maternidad respetuosa y multicultural en las unidades de neonatología.
\end{abstract}

Palabras-clave: Dinámica Poblacional, Maternidad, Recién Nacido Prematuro. 


\section{$\underline{\text { Resumo }}$}

Os processos de migração são um fenômeno global que pode resultar em condiçôes de vida mais vulneráveis. Perante estas condiçóes, torna-se relevante prestar o apoio necessário às famílias em situação de migração, em que alguns momentos críticos para isso são a gravidez, o nascimento e a criança. É assim que as mulheres migrantes vivenciam a maternidade em um contexto cultural diferente e, dadas as condiçôes desfavoráveis de vida, podem enfrentar dificuldades na gravidez, como o parto prematuro, o que pode representar uma experiência traumática e estressante. São poucos os estudos que abordam essas questôes na América Latina e, com isso, o objetivo deste estudo é descrever as experiências de mães migrantes com um filho ou filha prematuro internado numa unidade hospitalar de neonatologia. Foi utilizada uma metodologia qualitativa com abordagem fenomenológica. Foram realizadas entrevistas em profundidade com 16 mães, emergindo questóes como diferenças culturais sobre a maternidade e a criança, as condições de vida, as suas experiências no parto e, finalmente, o que significa ser mãe num país diferente do seu. Esses achados permitem uma melhor compreensão desse fenômeno e podem facilitar o desenvolvimento de estratégias que favoreçam uma maternidade respeitosa e multicultural nas unidades neonatais.

Palavras-chave: Dinâmica Populacional, Maternidade, Pré-termo.

\section{$\underline{\text { Abstract }}$}

Migration processes are a global phenomenon that can result in more vulnerable living conditions. Given these conditions, it is relevant to provide the necessary support for families in a situation of migration, in which pregnancy, childbirth, and child-rearing are some of the critical moments. This is how migrant women experience their motherhood in a different cultural context. Given the unfavorable living conditions, they may face difficulties in pregnancy such as premature birth, which could represent a traumatic and stressful experience. Few studies address these issues in Latin America and that is why the objective of this study is to describe the experiences of migrant mothers with a preterm son or daughter hospitalized in a neonatal unit. A qualitative methodology with a phenomenological approach was used. In-depth interviews were conducted with 16 mothers emerging relevant issues emerged such as cultural differences about motherhood and parenting, living conditions, their experiences in childbirth, and finally what it means to be a mother is in a different country than theirs. These findings allow a better understanding of this phenomenon and could facilitate the development of strategies that would promote respectful and multicultural motherhood in neonatal units.

Keywords: Population Dynamics; Maternity; Infant, Premature.

\section{Introducción}

La migración internacional es un fenómeno complejo y multifactorial relacionado con múltiples aspectos económicos, geopolíticos, sociales y culturales. En la actualidad 
los procesos migratorios son un fenómeno global y están presentes en gran parte del territorio mundial y afectan a todas las personas que habitan en ellos. Si bien las migraciones pueden representar diversas oportunidades para los países y las comunidades, también hay migraciones que ocurren en circunstancias más complejas, menos favorables y que resultan en condiciones de vida marcadas por mayores situaciones de vulnerabilidad (Organización Integral para las Migraciones, 2018). En los últimos ańos, se ha observado un aumento de las migraciones y los desplazamientos debido a diversos factores que van afectando la calidad de vida, tales como conflictos políticos e ideológicos, cambios en el medio ambiente y, falta de oportunidades y seguridad (Organización Integral para las Migraciones, 2018; Smith \& Floro, 2020).

Actualmente en Latinoamérica hay un importante movimiento migratorio en el cual Chile es considerado uno de los principales destinos para personas provenientes de diferentes países sudamericamericanos, al igual que de países de Centroamérica y otras partes del mundo, lo que ha generado un aumento en la población en situación de migración en el país (Stefoni, 2017). Según datos del Departamento de Extranjería y Migración (2018), Chile se ha ido convirtiendo paulatinamente en un país de destino de migración, donde en el año 2018 la cantidad de personas extranjeras residentes en Chile llegaba a un número aproximado de 1.251 .225 , donde el $52.6 \%$ corresponde a hombres y un 48,4 a mujeres, con una mayor concentración en el grupo etario entre los 20 y 30 ańos (59\%). Un informe realizado por la Comissão Econômica para a América Latina e o Caribe (2015) evidenció que entre los años 2010 y 2015 la inmigración en Chile creció en promedio un 4,9\% por año por sobre México y Brasil.

De acuerdo con el documento "Características de la Inmigración Internacional en Chile, Censo 2017-2019, del Instituto Nacional de Estadísticas”, la fecundidad de las mujeres migrantes ha ido tomando importancia conforme ha aumentado su llegada a Chile. Esto se ve reflejado en el incremento del porcentaje de partos de madres extranjeras respecto del total de nacimientos en los últimos ańos, donde estos partos representan un 7,9\% del total de nacimientos en Chile (Ojeda et al., 2018). Es de gran importancia prestar atención a los embarazos, partos y la crianza de los padres y madres que han migrado debido a la mayor situación de vulnerabilidad en la que se pueden encontrar, lo que podría afectar las trayectorias del desarrollo de la diada y de la familia. Un ejemplo de esto es que las madres migrantes pueden presentar un mayor riesgo de tener hijos/hijas prematuros/as (Bollini et al., 2009; Puthussery, 2016), lo que representa un inicio más complejo tanto para la madre como para el bebé debido a los riesgos neurobiológicos de la prematuridad (Aylward, 2014; Bilgin et al., 2018). En la actualidad no hay una gran cantidad de estudios que abarquen los temas relacionados con migración, embarazo y prematuridad en Latinoamérica, es por esto que se hace necesario conocer y comprender este fenómeno para poder desarrollar políticas necesarias para favorecer el acceso a los servicios que requieran las madres y sus bebés.

La compresión de la maternidad se ha ido modificando a lo largo de la historia, siendo ésta susceptible a los cambios sociales, políticos, económicas y culturales, y es así como este concepto puede ser considerado una construcción social y simbólica permeable a los cambios que van ocurriendo en el medio (Molina, 2006; Royo, 2011). Esta conceptualización puede ser modificada por las vivencias de las mujeres durante su desarrollo, el embarazo, el parto y el periodo posterior al nacimiento (CáceresManrique et al., 2014). Existen ciertos factores psicosociales que pueden tener un 
impacto en las experiencias de maternidad, tales como la presencia y características de las redes de apoyo social, el nivel ingreso económico, el tipo de trabajo, el nivel educacional de la madre y el acceso al sistema de salud (Benza \& Liamputtong, 2014; Gjerdingen et al., 2014; Jeong et al., 2013). Esta conceptualización de ser madre puede estar influenciada por las creencias personales, culturales y condiciones contextuales en las cuales vive la mujer (Benza \& Liamputtong, 2014), además, la vivencia de ser madre lejos de su país y cultura influirá de manera importante en su experiencia de maternidad (Higginbottom et al., 2014; Wikberg, 2020), y todo esto podría, no solo tener implicancias para la madre, sino que también para el desarrollo y bienestar de sus hijos/hijas.

A veces el periodo del embarazo puede ser complejo, debido a motivos económicos y laborales, por no poder acceder a los servicios que brindan cuidados prenatales, no conocer cómo funciona el sistema de salud y el desconocer temas propios del embarazo (Benza \& Liamputtong, 2014). Sin embargo, el hecho de ser madre migrante no condiciona necesariamente un factor de riesgo para la salud de la madre y del hijo/hija, no así, las condiciones de vida desfavorables que pueden presentar algunas mujeres en el nuevo país de destino (García-García et al., 2008; Miller et al., 2016). Esto, según algunos autores, puede conllevar a ciertos riesgos para la salud de la madre y del hijo/hija que está por nacer, dentro de estas problemáticas están los partos prematuros y el bajo peso al nacer (Bollini et al., 2009; Kartzow \& Castillo-Durán, 2012; Puthussery, 2016). En el caso de tener un parto prematuro, el recién nacido requerirá ser ingresado a la unidad de neonatología de un hospital, por lo que la experiencia de la maternidad se verá marcada por la temprana separación de su bebé, por la presencia de los monitores $\mathrm{y}$ artefactos de salud que producen una limitación en el contacto pero que son fundamentales para su sobrevida, por la imposibilidad de entregarle directamente los cuidados que requiere, y por las dificultades para acariciar y cargar a su bebé. A su vez esta realidad favorece la vivencia de emociones negativas y suele acompañarse de sintomatología depresiva y ansiosa lo que puede afectar el vínculo entre la díada madre e hijo (García et al., 2014; Jiménez et al., 2003; Lotterman et al., 2019; Trumello et al., 2018).

Por otra parte, se evidencia que el desarrollo de la maternidad dentro de la unidad de neonatología es un proceso complejo, debido a las dificultades en la interacción entre las madres y el bebé, a la transición lenta y paulatina hacia una mayor autonomía e independencia respecto a las decisiones y cuidados de su hijo/hija, y a las limitaciones para ejercer su rol de madre (Aagaard \& Hall, 2008; Ionio et al., 2017; Turner et al., 2015). Por tanto, las condiciones, vivencias y relaciones (padres-profesionales, padresbebé y profesionales-bebé) que se dan en este espacio determinarán de manera positiva o negativa la experiencia de la maternidad. Si a esto se le añade tener una escasa red de apoyo, no compartir la misma cultura, y las barreras que existen para el acceso a la información y a los servicios públicos, la diada se encontrará en una situación compleja y de mayor riesgo (Kartzow \& Castillo-Durán, 2012). Otro aspecto que puede condicionar la salud de la madre y la vivencia de la maternidad son las barreras idiomáticas, ya que dificultan la comunicación y relación entre madre y equipo de salud (Fair et al., 2020; Sánchez-Fernández, 2003).

El parto prematuro es considerado como una experiencia estresante y traumática para los padres (Brunson et al., 2021; Nix \& Ansermet, 2009), es así como numerosos 
estudios han abordado el estrés que estos viven durante el periodo de hospitalización de su hijo prematuro (Ho et al., 2010; Ionio et al., 2019; Shaw et al., 2006). Dentro de la estadía en el hospital, los padres no solo deben lidiar con estresores propios del ambiente de las unidades de neonatología, sino que también deben enfrentar las complicaciones o incertidumbres respecto al estado de salud de su hijo, de la separación física y emocional con su bebé, y del estrés propio de la parentalidad (Shaw et al., 2006; Dudek-Shriber, 2004). Las madres de infantes nacidos de pretérmino evidencian una tasa de depresión post parto de casi el doble que las madres de bebés de término (28\% - 40\%), especialmente en el periodo temprano de postparto cuando aún están en las unidades de neonatología (Fredriksen et al., 2019). Estos datos son de relevancia debido al impacto que puede tener un parto prematuro y la hospitalización de su bebé para la salud mental de las madres en situación de migración.

Dada la realidad chilena en cuanto a los procesos de migración y las probabilidades que debido a las condiciones de vida y las dificultades del acceso a la salud las mujeres migrantes tengan un parto prematuro, es que nace el interés de conocer las experiencias de las madres migrantes en una unidad de neonatología, siendo este un espacio donde sus bebés deben estar hospitalizados como parte de las prestaciones de salud que entrega el Estado. Surge de esta forma la pregunta respecto a cómo viven las madres en situación de migración la experiencia de tener un bebé prematuro que debe ser hospitalizado. El objetivo de este estudio es conocer y describir las experiencias de madres migrantes con un hijo o hija de pretérmino hospitalizado/a en una unidad de neonatología de un hospital público de la ciudad de Santiago de Chile. De este modo se podrá enriquecer el conocimiento disponible y así responder de una mejor manera a las necesidades de una maternidad multicultural en un contexto tan particular como lo son estas unidades de atención.

\section{Método}

\section{Participantes}

Las participantes de este estudio fueron 16 mujeres en situación de migración cuyos hijos/as nacieron prematuros y se encontraban hospitalizados en el Servicio de Neonatología de un hospital público de la ciudad de Santiago, Chile. Dentro de los criterios de inclusión están ser mayor de 18 años, ser madres en situación de migración, hablar español fluido y tener un hijo o hija de pretérmino hospitalizado en la unidad de neonatología. Como criterio de exclusión, se encontraba el que la madre presentara consumo problemático de alcohol o drogas, o algún trastorno psiquiátrico grave como esquizofrenia (psicosis). La estrategia de muestreo utilizada corresponde a una metodología no probabilística por conveniencia, donde la selección de los sujetos del estudio dependió de ciertas características o criterios relacionados con el objeto de estudio (Otzen \& Manterola, 2017), lo que en este caso sería ser mujer en situación de migración y ser madre de un bebé de pretérmino hospitalizado. El tamaño muestral fue definido a través del criterio de saturación de contenido. Se realizó una invitación 
a participar a las madres por lo menos una semana después del ingreso a la unidad de neonatología, tomando los resguardos éticos necesarios para proteger a las diadas durante todo el proceso del estudio.

\section{Diseńo del estudio y procedimientos}

Esta investigación se realizó a través de una metodología cualitativa, la cual nos permitió obtener y analizar las narrativas de las madres de bebés prematuros, otorgándole importancia a las experiencias subjetivas de las participantes en relación con sus vivencias sobre la maternidad. Para este estudio se utilizó un enfoque Fenomenológico, ya que las premisas de éste buscan comprender la realidad desde la perspectiva de los mismos sujetos, para así poder explicar la naturaleza y características de los fenómenos (Martínez, 2012). De esta forma podríamos acercarnos a una mejor comprensión del fenómeno migración, maternidad y prematuridad, evitando describir o comprender este fenómeno desde la única perspectiva de los investigadores, desde sus propios preconceptos y subjetividades, y no desde la vivencia de las personas que están esa realidad particular.

Los evaluadores realizaron entrevistas en profundidad con todas las participantes de manera individual cuando sus bebés aún permanecían hospitalizados. Estas se efectuaron fuera de las unidades de neonatología para favorecer un espacio de tranquilidad y confort para las madres. Esta técnica de producción de información nos permitió acceder a aspectos de profundidad asociados a las valoraciones, motivaciones, deseos, creencias y esquemas de interpretación de los participantes (Cerón, 2006).

Las entrevistas fueron realizadas entre los años 2017 y 2019. Éstas fueron almacenadas a través de grabación de voz, teniendo una duración de aproximadamente una hora y posteriormente fueron transcritas por el equipo investigador.

\section{Consideraciones éticas}

Este estudio contó con la aprobación del Comité de Bioética de la Universidad Andrés Bello. Todos los participantes invitados consintieron su participación en el estudio, dejándolo estipulado en el consentimiento informado.

\section{Análisis de datos}

Para el análisis de la información recopilada se utilizó la técnica de análisis de contenido, ésta nos permite analizar las narrativas de las madres entrevistadas en relación al contexto del cual surgen, y de esta forma interpretarlas y generar un nuevo conocimiento (Assarroudi et al., 2018; Bardin, 1996; Roller, 2019). Es así como se van transformando los relatos textuales no estructurados en unos más estructurados y organizados para poder interpretarlos, de está forma van que emergiendo las unidades de análisis con significado particular que se denominan categorías (Parra et al., 2017). Para llevar a cabo lo anterior, se transcribieron las entrevistas y para cada una se realizó un análisis de contenido por dos evaluadores independientes. Luego ambos investigadores llegaron a un consenso con sus análisis e integraron las 16 matrices de 
análisis individual en una matriz grupal, proceso por el cual se pudo obtener las categorías emergentes finales.

\section{Resultados}

En este estudio participaron 16 madres en situación de migración provenientes de diversos países, tales como Perú, Venezuela, Colombia entre otros (ver Tabla 1). La edad de las madres fluctuaba entre los 22 y 44 años $(M=32,60 ; D S=5,76)$ y la edad gestacional de sus bebés al nacer se encontraban en el rango de las 27 y 36 semanas $(M=31,90 ; D S=3,24)$. El tiempo que llevan en Chile va desde los 7 meses hasta los 3 años, todas residen en la ciudad de Santiago y se encontraban en trámite para su residencia definitiva en Chile. Las participantes de este estudio vivían con sus parejas o familia nuclear, donde todos los miembros del grupo estaban en situación de migración. La participación laboral era variada, donde algunas de ellas estaban con contratos formales y otras participaban en trabajos remunerados informales.

Tabla 1. Características de los participantes.

\begin{tabular}{ccccccc}
\hline Identificación & Nacionalidad & $\begin{array}{c}\text { Edad } \\
\text { (ańos) }\end{array}$ & Estado civil & $\begin{array}{c}\text { Primeriza } \\
\text { Sí/No }\end{array}$ & Paridad & $\begin{array}{c}\text { Parto } \\
\text { (semanas) }\end{array}$ \\
\hline M1 & Venezolana & 30 & casada & Sí & 1 & 36 \\
\hline M2 & Peruana & 32 & soltera & Sí & 1 & 31 \\
\hline M3 & Peruana & 30 & soltera & No & 1 & 34 \\
\hline M4 & Rusa & 36 & soltera & Sí & 1 & 35 \\
\hline M5 & Haitiana & 25 & soltera & No & 2 & 35 \\
\hline M6 & Cubana & 34 & casada & Sí & 2 & 29 \\
\hline M7 & Peruana & 33 & con pareja & No & 1 & 29 \\
\hline M8 & Peruana & 35 & casada & Sí & 1 & 27 \\
\hline M9 & Ecuatoriana & 44 & con pareja & No & 1 & 36 \\
\hline M10 & Venezolana & 23 & soltera & Sí & 1 & 29 \\
\hline M11 & Venezolana & 35 & casada & Sí & 1 & 32 \\
\hline M12 & Colombiana & 22 & con pareja & No & 1 & 29 \\
\hline M13 & Peruana & 31 & soltera & No & 2 & 32 \\
\hline M14 & Venezolana & 36 & soltera & Sí & 1 & 27 \\
\hline M15 & Colombiana & 39 & separada & No & 1 & 33 \\
\hline M16 & Peruana & 35 & con pareja & No & 1 & 36 \\
\hline
\end{tabular}

A partir de las narrativas de las madres emergieron 5 categorías con sus respectivas subcategorías. Éstas dan a conocer los aspectos significativos que se relacionan con las vivencias de las madres en situación de migración que han tenido un bebé prematuro que ha sido hospitalizado en una unidad de neonatología (Tabla 2). 
Tabla 2. Categorías y subcategorías emergentes.

\begin{tabular}{|c|c|}
\hline Categorías emergentes & Subcategorías emergentes \\
\hline \multirow{2}{*}{ 1. Comprensión de la maternidad y crianza } & 1.1 Comprensión de la maternidad \\
\hline & 1.2 Diferencias culturales en relación a la crianza \\
\hline \multirow{4}{*}{ 2. Maternidad, migración y prematuridad } & $\begin{array}{l}\text { 2.1 Nacimiento de su bebé en país diferente al de } \\
\text { origen de la madre }\end{array}$ \\
\hline & 2.2 Experiencia del parto \\
\hline & 2.3 Condiciones de vida de las madres migrantes \\
\hline & 2.4 Expectativas en relación a alta del hospital \\
\hline \multirow{4}{*}{ 3. Ser madres en una unidad de neonatología } & 3.1 Rol de madre en la unidad de neonatología \\
\hline & $\begin{array}{l}3.2 \text { Interacciones con sus bebés dentro de la unidad } \\
\text { de neonatología }\end{array}$ \\
\hline & $\begin{array}{l}\text { 3.3 Espacios de participación de las madres } \\
\text { migrantes dentro del Servicio de Neonatología }\end{array}$ \\
\hline & 3.4 Experiencias en la unidad de neonatología \\
\hline \multirow{2}{*}{ 4. Experiencias emocionales de las madres } & $\begin{array}{l}\text { 4.1 Sentimientos y emociones que experimentan las } \\
\text { madres migrantes }\end{array}$ \\
\hline & $\begin{array}{l}\text { 4.2 Preocupaciones de las madres migrantes a por la } \\
\text { fragilidad de sus bebés }\end{array}$ \\
\hline \multirow{3}{*}{$\begin{array}{l}\text { 5. Factores que facilitan u obstaculizan sus } \\
\text { experiencias en la unidad de neonatología }\end{array}$} & 5.1 Rol del equipo de salud \\
\hline & $\begin{array}{l}\text { 5.2 Experiencia de las madres en el sistema de salud } \\
\text { chileno }\end{array}$ \\
\hline & 5.3 Red de apoyo social \\
\hline
\end{tabular}

\section{Comprensión de la maternidad y crianza}

La primera categoría hace referencia a la construcción de la comprensión de maternidad y de la crianza de los niños y niñas, las cuales se ven influenciadas por sus creencias, cultura y por las vivencias personales de cada una. Es así como la primera subcategoría se relaciona con lo que las mujeres migrantes de este estudio refieren en torno a la maternidad, lo que se puede observar en los siguientes discursos, "Siempre cambian algunas cosas, cada país tiene sus creencias y en torno a eso cambia la maternidad" (M13), “...las madres son internacionales (...) el fondo no cambia, la forma es la que cambia, pero el objetivo no creo que cambie, son madres donde quieras" (M6). Donde se puede extraer la idea de que si bien puede haber una compresión común sobre este constructo hay matices que van diferenciando la concepción de maternidad en las distintas culturas.

La segunda subcategoría muestra las diferencias en cuanto a las creencias sobre la crianza, lo que se plasma en los siguientes extractos de las entrevistas "El rol de ser mamá acá es prioridad... el hijo es prioridad, allá no te incentivan a ser mamá (haciendo referencia a su país de origen)... las abuelas se hacen cargo de los niños"(M7), "Las mamás chilenas son muy consentidoras con los niños... en cambio nosotras corregimos muchos más (...), no cargamos mucho a los bebés ... hay un apego más distante” (M9), estas palabras denotan como el país en el que residen actualmente tiene otra concepción sobre los cuidados que se les deben dar a los bebés, en cuanto a quienes ejercen el rol de cuidadores 
principales y en cómo deben relacionarse con sus hijos/as. Es así como estas diferencias en torno a la crianza pueden tensionar las relaciones entre las madres y el equipo de salud: "... tiene que ser como ellos dicen...cuando yo trato de calmar a mi hija en la cuna, ellos quieren que tu la saques y la calmes en brazos, entonces eso te choca" (M10), "llega la matrona y me dice que asi no es lo correcto, que no tengo que tocarlo asi, en cambio con mi otra hija yo la tocaba con mi estilo y con mi familia... acá hay poca privacidad y te limitan" (M7).

\section{Maternidad, migración y prematuridad}

La segunda categoría revela temas relacionados con la migración, la maternidad y el tener un hijo/a prematuro/a. Uno de los temas que emergió fue el hecho de que su hijo/hija naciera en un país diferente a su país de origen, los discursos en este caso se centran en lo favorable que ha sido en relación a la sobrevida y bienestar de su bebé, lo que se puede desprender de los siguientes relatos. "Acá los niños están súper protegidos..."(M8), "Si hubiese nacido en mi país habría sido terrible por la situación actual. Yo creo que mi hija se habría muerto con el primer bajón de luz..." (M10), "si hubiese nacido en mi país la historia sería otra... mi hijo hubiese muerto" (M14). Estos últimos relatos se pueden relacionar con la situación sanitaria actual de su país de origen, donde ellas visualizan una mayor vulnerabilidad y escases de recursos.

A pesar de que estos relatos evidencian que ellas perciben que sus hijos/as están más protegidos en este contexto de salud, las experiencias de parto fueron más bien traumáticas, no obstante, esto puede deberse a lo complejo y estresante que es un parto prematuro. Las difíciles experiencias de parto las podemos ver reflejadas en las palabras de las madres, "fue traumático... no esperaba que naciera" (M8), "Fue horrible, me quería como morir...incluso los dos primeros dias yo no pude bajar a ver a la niña (...) es angustiante, sentir un vacio porque no tienes nada dentro de ti y no tienes nada afuera” (M10). "...ese día no paré de llorar, no lo pude mirar porque la metieron en su bolsita para meterlo en su incubadora" (M14). Estos fragmentos pueden ser un reflejo del trauma vivenciado en el momento del parto y los primeros días posterior a este evento, lo que se puede transformar en otra variable que puede impactar en el bienestar emocional de la madre y el bebé.

La tercera subcategoría emerge en relación a la condiciones de vida de las madres en situación de migración, las cuales en algunos casos pueden complejizar tanto las visitas a las unidades de neonatología como la preparación de un lugar para vivir con bebé luego del alta. Esto lo podemos observar en las siguientes narrativas, "Me pasa con el tema de la casa, para que den de alta a mi bebé tengo que tener la casa bonita, una pieza adecuada, estoy tratando de hacerlo, pero afuera no tengo apoyo económico"(M7), "No ha sido el mejor momento para tener a mi bebé por el tema de la economía... el tema económico es lo difícil para uno que está en otro país, no sé qué haremos con los gastos de mi hijo cuando salgamos de acá" (M9), "A veces se me dificulta venir, a veces no puedo venir por la parte económica" (M12). Es así como las condiciones socioeconómicas y la falta de redes de apoyo de las madres van generando tensión y estrés en relación a los cuidados y necesidades que deberán solventar para mantener a sus bebés.

A pesar de las dificultades que las madres deben enfrentar en su cotidianidad ellas tienen expectativas positivas frente al alta de las unidades de neonatología. "Me imagino 
que va ser el momento más feliz al llevarlo a la casa y que él esté bien, asi estaré todo el tiempo con él y lo podre cuidar” (M12), “...yo sé que el día que ellas salgan de acá... voy a estar feliz porque sé que van a estar conmigo, no tendré que venir hasta acá...”(M8). Ellas añoran el día que puedan llevar a los bebés a sus hogares y poder vivenciar en plenitud la maternidad, en un espacios que a pesar de representar algunas carencias o necesidad, estos constituyen su hogar y es donde quieren estar con su familia.

\section{Ser madres en una unidad de neonatología}

La maternidad para estas mujeres implica un cambio relevante en sus vidas, representada por una importante entrega y preocupación por su bebé, siendo una experiencia única, que solo se comprende a cabalidad cuando se experimenta y que pareciera ser sentida por la mayoría de las madres entrevistadas de una forma similar. A través de los discursos emergió la primera subcategoría que se relaciona con cómo las mujeres van desarrollando su rol de madre en las unidades de neonatología, que a veces toma una forma particular por las circunstancias en las que se encuentran. Esto se refleja en los siguientes extractos de las entrevistas: “...desde que yo llego quiero tomarlo, tenerlo en mis brazos...no soltarlo y estar pendiente" (M7), "...abrazarlo, uno le conversa, le habla, él te mira, escucha (...) lo más importante es darle cariño, darle el amor que no se sienta solo" (M3). Estas palabras muestran esta necesidad de contacto, de interacción y de evitar que su bebé experimente la soledad, aspectos que ellas mismas pueden haber experimentado cuando llegaron a este país que era nuevo y desconocido para ellas. Otros relatos de las madres se centran más en las limitaciones para poder ejercer su rol, "No le he podido cambiar ni su primer pañal" (M1), "El mes anterior estuvieron en incubadora entonces eso es siempre una barrera, es una barrera real física que está" (M6), "No lo he podido desarrollar mucho (haciendo referencia al rol de madre), porque en realidad no tienes el tiempo suficiente... el tiempo se pasa muy rápido, por ejemplo mi niña está en incubadora y hay que esperar que la valoren y después te la pasan... no tienes mucho tiempo" (M9). Estas barreras físicas o temporales van limitando el deseo que ellas tienen de relacionarse y cuidar a sus hijos/as.

Las interacciones entre la madre y su bebé son fundamentales ambos y éstas van tomando forma entre las características de ambos y las condiciones del contexto. A través de las narrativas de las madres podemos conocer un poco más acerca de estas interacciones y del vínculo que van formando, "le canto, lo puedo acariciar, le digo mucho que lo amo y él me levanta la cabeza y me mira... hay un vínculo, el sabe quien es su mamá y lo puedo sentir" (M8), "Ahora yo puedo tocarlo, comunicarme con él y tomarlo en brazos" (M4). Estas palabras reflejan como ellas sienten que van forjando un vínculo afectivo con sus hijos/as y que esta relación es bidireccional debido a que sus bebés responden con sus miradas o movimientos. Sin embargo, las limitaciones en el horario de visita y el tener que separarse de sus bebés tiene un impacto negativos para ellas, "Es emocionalmente horrible, porque tu sientes un vacio cuando te vas a casa todas las noches" (M10), "Estás todo el día en el hospital, pero no todo el día con tu niña, considero que es muy poco tiempo (haciendo referencia al tiempo que puede estar con su hija)"(M9). Esto puede generar emociones negativas porque ven limitado el tiempo y espacio para la interacción con sus hijos/as. 
Las madres expresan su necesidad de tener mayores espacio de participación en relación a los cuidados de sus bebés, lo que se puede extraer de los siguientes relatos, "Que dejen pasar más tiempo, que dejaran que uno tuviera más contacto con su hijo" (M1) "... que te dejen cambiar a ti el pañal..." (M2), "El biberón se lo da otra persona, me impresiona que esté sentado... es falta de apego entiendo... ojalá pudiera alimentarlo" (M11), “...me gustaría tener una mayor libertad para verlo y sentirlo” (M 15). Tener mayores espacios de participación podría facilitar el proceso de transición del hospital a los hogares de las familias, además de favorecer el vínculo, y la confianza en ellas como madres.

La última subcategoría describe cómo estas mujeres han percibido el estar dentro de una unidad de neonatología con sus bebés, esto quedó plasmado en los relatos de las madres, "Mi experiencia... fue bastante trágica. Estar aqui todos los días es agotador... el hospital es agotador. Estos han sido los 67 días más largos de mi vida, es terrible porque tú vienes acá y no sabes que te espera...siempre estás en el desierto a la deriva de lo que pase" (M10), "... el día a día es como una montaña rusa de emociones... vienes con la expectativa de que algo puede pasar" (M11), "El ruido de las alarmas y las restricciones me desesperan" (M15). Estas narrativas nos muestran lo relevante que es abordar estas temáticas para que las experiencias de las madres sean más positivas y menos traumáticas, y así favorecer el bienestar de esta diada.

\section{Experiencias emocionales de las madres}

En este contexto surgen diferentes sentimientos y emociones experimentados por las participantes que complejizan la vivencia de la maternidad. “...llegó a la casa y veo sus fotos y me pongo a llorar... me da pena..." (M16); “...me siento, asi como triste, pero igual tengo que tirar para arriba" (M3); "La experiencia es un poco dura... que sean tus primeros hijos y justo prematuros... tengo mucho miedo porque... los encuentro tan frágiles" (M6), "... el día a día es como una montaña rusa de emociones... vienes con la expectativa de que algo puede pasar" (M11), "Sentía como un infarto, nunca voy a a olvidar esa sensación terrible (refiriéndose al primer día que estuvo en la unidad de neonatología). La sensación que me produce la incubadora es la misma que me produce un ataúd... no me gusta ver que mi niño está tan quieto" (M15).

Como lo evidencia la segunda subcategoría muchos de estos aspectos emocionales se vinculan a la fragilidad que ellas perciben de sus bebés. "... es mucho más chiquitito, da como miedo moverlo, es tan indefenso, es diferente”(M7), “... verlo tan pequeñito y tan frágil fue muy difícil”. Esto también se ve reflejado en los temores que tienen hacia el futuro, "Mi principal temor de que haya sido prematura es que pasemos toda la vida metidas en un hospital... que le genere muchos problemas en su crecimiento" (M10).

\section{Factores que facilitan u obstaculizan sus experiencias en la unidad de neonatología}

Los temas que emergieron en relación a esta categoría son de gran relevancia dado que nos permitirían identificar aspectos que deberían ser trabajados para favorecer una mejor experiencia de las madres en las unidades de neonatología. En relación a esto el equipo de profesionales de la salud que trabajan en las unidades de neonatología tendrían un rol fundamental, lo que se puede observar en los discursos de las madres, 
"Me tranquiliza saber que mi bebé está en buenas manos... han sido muy amables" (M1), "se ve un interés profesional... y la niña que me pasó a mi bebé fue muy amable y me explicó todo sobre la prematuridad, me dio mucho apoyo ... me han entregado siempre información clara" (M9). Relatos como estos evidencian que este tipo de acciones pueden facilitar que la madre pueda lidiar de mejor manera los estresores de estos contextos.

No obstante, hay acciones del equipo de salud que pueden afectar negativamente a las madres y estas fueron descritas por madres migrantes que eran más jóvenes. Esto se puede desprender de los siguientes relatos, "Hay algunos doctores que te tratan mal, no te tienen paciencia ... no se ponen en tu posición" (M7), "Yo diría que no habia mucha sensibilidad en el momento de tratar con el paciente" (M6), "... el personal es muy bueno, está muy capacitado, pero hay veces que te tratan muy mal. Te contestan mal, te echan, te tratan como si no supieras nada" (M10). "De repente ustedes como profesionales son muy desapegados... parece que no les importa...aunque si hay personas que logran ayudar" (M14). Estos relatos muestran aspectos duros, poco humanizados y poco empáticos en la interacción entre el profesional de la salud y las madres, lo que puede impactar negativamente en la salud mental de ellas y en la confianza que van desarrollando hacia quienes están cuidando a sus bebés.

En cuanto a las experiencia de las madres en el sistema de salud chileno podemos ver que hay diversas experiencias, desde las que perciben que en este país hay un mayor resguardo para las embarazadas y los niños y niñas, como las que aún no conocen bien cómo funciona el sistema de salud. "Acá es como más cuidado... la embarazada es prioridad” (M7), “...no hay diferencias si eres extranjero, te atienden de la misma manera, te dan los mismos beneficios (en relación a los beneficios del Estado para embarazadas y los recién nacidos)" (M10), "...las leyes de acá no las conozco mucho, pero las estoy conociendo" (M9). Relatos que nos hacen darnos cuenta de que cada madre vivencia esto de forma diferente, lo que se puede relacionar con diversos factores que fueron marcando sus experiencias (por ejemplo, el acceso a la información, comprensión de la información, entre otros), y que puede influenciar la relación futura que esta madre mantendrá con el sistema de salud (asistencia a controles, acceso a los programas estatales para los infantes, etc).

La última subcategoría hace referencia a las redes de apoyo social las que son esenciales para resguardar el bienestar de las madres y de sus bebés.. En las entrevistas fueron apareciendo ideas que se conectaban con la añoranza de estar con sus familias o cercanos, "... no tienes a nadie que te pueda ayudar... el dolor es el mismo... pero cuando uno tiene a la familia uno lo canaliza mejor" (M8), "Ha sido terrible para mi como persona, porque estoy lejos de todo, de todo mi entorno" (M10). estas palabras denotan la importancia de la cercanía de la familia en este contexto. Otras madres hacían referencia al apoyo que reciben de sus parejas "... habia momentos que ya no daba para más pero mi esposo siempre es positivo" (M7), "En mi experiencia con mi pareja nos ayudamos bastante... estamos unidos, sabemos lo que tenemos que hacer por nuestro bebé y por nosotros" (M13). Entre las madres se ha creado una importante fuente de apoyo y fortalecimiento, donde van compartiendo experiencias y van recibiendo a las madres que van llegando, facilitando el que puedan comprender cómo es ser madre en un servicio de neonatología "... tengo el apoyo entre nosotras que estamos en lo mismo... hemos hecho un grupo, entre nosotras nos fortalecemos" (M7). 


\section{Discusión}

A través del análisis de las entrevistas se buscó responder al objetivo de este estudio el cual era conocer y describir las experiencias de madres migrantes con un hijo o hija de pretérmino hospitalizado/a en una unidad de neonatología. Es así como fue emergiendo información relacionada con las vivencias de las madres migrantes en este contexto, lo que fue enriqueciendo la comprensión de este fenómeno.

En las narrativas de las mujeres en situación de migración aparecen las diferencias culturales en cuanto a la comprensión de la maternidad y de las prácticas de crianza. Esto es importante de considerar debido a que las madres migrantes tendrán desafíos particulares en su maternidad y crianza por estar inmersas en un ambiente cultural con valores y creencias que pueden ser diferentes a las de su cultura de origen (Cheah et al., 2013). De esta forma, el proceso de aculturación en relación con estos aspectos puede ser una fuente de estrés familiar que podría impactar directamente las actitudes de crianza en los padres migrantes (Kiang et al., 2017; Telzer, 2010). Estas diferencias culturales deberían ser consideradas en el diseño e implementación de las políticas de salud tanto a nivel prenatal como postnatal para los padres y sus bebés (Savage et al., 2007), para facilitar el acceso y participación de éstos en las prestaciones que son parte de los programas del Estado, evitando los prejuicios frente a las creencias o actitudes de los cuidadores.

A pesar de las complejas condiciones de vida que pueden tener las madres migrantes, en este estudio, todas las madres hacen referencia a que el bienestar y salud de sus bebés es mejor que si hubieran nacido en su país de origen, lo que coincide con lo descrito en la literatura, donde se señala que a pesar de las dificultades socioeconómicas y culturales de las poblaciones en situación de migración éstas podrían tener mejores niveles de salud que en el país de donde provienen (Miller et al., 2016). Es por esto que toma más relevancia conocer las experiencias de las madres en las unidades de neonatología y determinar cuales son los aspectos que ellas consideran como facilitadores $\mathrm{u}$ obstaculizadores. En las entrevistas se observó que una relación, trato y actitud positiva, al igual que el profesionalismo del equipo de salud entregan confianza y tranquilidad a las madres, configurándose como un aspecto fundamental que se constituye como factor protector dentro de su estadía en el hospital. Esto concuerda con los resultados del estudio de CanoMoroba et al. (2012), los cuales vinculan las relaciones entre las madres y el equipo de salud como facilitador de la experiencia dentro de las unidades de neonatología.

En cuanto a la calidad y valoración de la atención que reciben en las unidades de neonatología, esta estaría estrechamente relacionada con las experiencias y las relaciones que se establecen entre las madres y los profesionales del equipo de salud, constituyéndose como factor fundamental que tiene un impacto directo en las experiencias de dichas madres, lo cual concuerda con el estudio de Aagaard \& Hall (2008) que destaca como un aspecto relevante la relación que se establece entre las madres y las enfermeras durante la hospitalización. No obstante, las madres de este estudio señalan que uno de los factores que impactó de forma negativa su experiencia fue la falta de información entregada y el trato duro y poco empático que recibieron, lo que concuerda con lo observado en otros estudios, los cuales además hacen énfasis en que estos aspectos deben ser incorporados en el trabajo de los equipos de las unidades 
de neonatología (Kasat et al., 2020; Pham et al., 2018). Del mismo modo la investigación de Rossel et al. (2002), destaca la necesidad de facilitar la comunicación de la madre con el equipo de salud, dando énfasis a las verdaderas necesidades de las madres, y a la importancia de responder con actitudes y gestos que sean empáticos y contenedores. Debemos considerar que la comunicación puede ser compleja en grupos multiculturales, produciéndose frustraciones en los procesos comunicativos entre las personas (Ramos-Vidal, 2011) y esto es algo que deben tener en cuenta los equipos de salud.

En cuanto al rol de madre y a los espacios de participación, las madres señalan que a pesar de pasar largos periodos de tiempo en el hospital, el horario de visita es muy reducido y que es muy poco lo que ellas pueden hacer en relación a los cuidados de sus bebés, como por ejemplo, alimentarlos. No obstante, la literatura sostiene que el brindar mayores tiempos y espacios de participación e interacción con sus bebés tendría un efecto positivo tanto a nivel de salud mental y satisfacción parental de las madres como para el bienestar de los bebés (Harding et al., 2019; Liu et al., 2018). Estos espacios serían cruciales para la relación entre la madre y el bebé, donde estos se irían retroalimentando mutuamente y fortaleciendo su vínculo (Koroleff, 2001).

Es importante considerar que las narrativas de las madres responden a una compleja construcción entre sus propias vivencias como hijas y mujeres en una cultura y grupo social en el cual fueron criadas, sus experiencias de vida como personas en situación de migración y el hecho de haber enfrentado un parto prematuro y los primeros momentos de cuidado de sus bebés en un ambiente tan ajeno como las unidades de neonatología. Si bien puede haber tensiones y problemáticas propias de la migración, lo que resalta en sus palabras es la preocupación por la fragilidad de sus bebés, evidenciando estados emocionales más negativos y requiriendo de la contención de un equipo de salud que sea cálido, empático y que mantenga una comunicación continua con las familias. Esto puede ser explicado por lo que Stern (2004) llama la Constelación Materna, donde las preocupaciones de las madres están centradas en la preocupación por el desarrollo y crecimiento de su bebé y en los cambios que ella va vivenciando como madre. Sin embargo, siempre debemos estar atentos a sus necesidades porque en la transición al hogar pueden resurgir los temas ligados a la migración y al encuentro con una cultura que comprende la maternidad y la crianza de una forma diferente.

Esta investigación representa un acercamiento al entendimiento de cómo viven las madres en situación de migración la experiencia de tener un bebé prematuro que debe ser hospitalizado, lo que significa un aporte a la comprensión de este fenómeno desde las propias narrativas de las madres. Esto sin duda responde la pregunta de cómo fueron las vivencias durante la hospitalización de sus bebés para este grupo de mujeres y nos abre un camino con nuevas interrogantes para ser abordadas en futuras investigaciones. El fenómeno de la migración internacional es algo complejo y multifactorial, y si bien en este estudio participaron dos mujeres que tenían un hijo en su país de origen a cargo de sus abuelos, en este artículo no se ahondo en estas relaciones, no obstante, si se reconoce como esta maternidad transnacional impacta su maternidad actual. Se debe continuar estudiando esto en poblaciones más diversas y ahondar con mayor profundidad en las historias y necesidades de las madres migrantes, en cuanto a su pasado, su presente y cómo proyectan su futuro, para así junto a ellas co-construir espacios de participación y de un mejor acceso a la salud. 
Si bien el fenómeno de la migración internacional es algo complejo y multifactorial, como fortaleza de este estudio podemos mencionar que esta investigación representa un acercamiento al entendimiento de cómo viven las madres en situación de migración la experiencia de tener un bebé prematuro que debe ser hospitalizado, lo que significa un aporte a la comprensión de este fenómeno desde las propias narrativas de las madres. Esto sin duda responde la pregunta de cómo fueron las vivencias durante la hospitalización de sus bebés para este grupo de mujeres y nos abre un camino con nuevas interrogantes para ser abordadas en futuras investigaciones. No obstante, hubo elementos que no fueron abordados, por ejemplo, en este estudio participaron dos mujeres que tenían un hijo en su país de origen a cargo de sus abuelos, y que, si bien en este artículo no se ahondo en estas relaciones, si se reconoce como la maternidad transnacional impacta su maternidad actual. Sumado a esto sería relevante contar con las narrativas de madres que no manejan el español de forma fluida, a través del apoyo de interpretes, lo que nos permitirían tener mayor conocimiento del impacto de la migración en la maternidad. Se debe continuar estudiando esto en poblaciones más diversas y ahondar con mayor profundidad en las historias y necesidades de las madres migrantes, en cuanto a su pasado, su presente y cómo proyectan su futuro, para así junto a ellas co-construir espacios de participación y de un mejor acceso a la salud. En esto todos tenemos un rol crucial, desde los profesionales que trabajan en las unidades de neonatología (médicos, enfermeras, terapeutas ocupacionales, kinesiólogos/fisioterapeutas, fonoaudiólogos/terapeutas de lenguaje, técnicos, entre otros) como quienes brindan apoyo luego del alta, y también quienes trabajan en gestión y políticas públicas en salud.

\section{Agradecimientos}

Al Servicio de Neonatología del Hospital Luis Tisné y a Fernanda Alamos, Isidora Barañao, María José Larraín, Camila Palma, María Paz Peña y Valentina Riveros.

\section{Referencias}

Aagaard, H., \& Hall, E. O. C. (2008). Mothers' experiences of having a preterm infant in the neonatal care unit: a meta-synthesis. Journal of Pediatric Nursing, 23(3), 26-36. http://dx.doi.org/10.1016/j.pedn.2007.02.003.

Assarroudi, A., Nabavi, F. H., Armat, M. R., Ebadi, A., \& Vaismoradi, M. (2018). Directed qualitative content analysis: the description and elaboration of its underpinning methods and data analysis process. Journal of Research in Nursing, 23(1), 42-55.

Aylward, G. P. (2014). Neurodevelopmental outcomes of infants born prematurely. Journal of Developmental and Behavioral Pediatrics, 35(6), 394-407. http://dx.doi.org/10.1097/01.DBP.0000452240.39511.d4.

Bardin, L. (1996). Análisis de contenido. Madrid: Akal.

Benza, S., \& Liamputtong, P. (2014). Pregnancy, childbirth and motherhood: a meta-synthesis of the lived experiences of immigrant women. Midwifery, 30(6), 575-584.

Bilgin, A., Mendonca, M., \& Wolke, D. (2018). Preterm birth/low birth weight and markers reflective of wealth in adulthood: a meta-analysis. Pediatrics, 142(1), e20173625. http://dx.doi.org/10.1542/peds.2017-3625.

Bollini, P., Pampallona, S., Wanner, P., \& Kupelnick, B. (2009). Pregnancy outcome of migrant women and integration policy: a systematic review of the international literature. Social Science \& Medicine, $68(3), 452-461$. 
Brunson, E., Thierry, A., Ligier, F., Vulliez-Coady, L., Novo, A., Rolland, A. C., \& Eutrope, J. (2021). Prevalences and predictive factors of maternal trauma through 18 months after premature birth: a longitudinal, observational and descriptive study. PLoS One, 16(2), 1-16. http://dx.doi.org/10.1371/journal.pone.0246758.

Cáceres-Manrique, F. M., Molina-Marín, G., \& Ruiz-Rodríguez, M. (2014). Maternidad: un proceso con distintos matices y construcción de vínculos. Aquichan, 14(3), 316-326. http://dx.doi.org/10.5294/aqui.2014.14.3.4.

CanoMoroba, S., Marselliés, M. A., \& Jové, G. (2012). Un paseo por la $5^{\mathrm{a}}$ planta. Análisis de las vivencias y el grado de satisfacción de los padres de bebés hospitalizados en la UCIN del hospital universitario Arnau de Villanueva de Lleida. Revista de Educación Inclusiva, 5(3), 23-34.

Cerón, M. C. (2006). Metodologías de la investigación social. Santiago: LOM ediciones.

Cheah, C. S., Leung, C. Y., \& Zhou, N. (2013). Understanding “tiger parenting” through the perceptions of Chinese immigrant mothers: Can Chinese and US parenting coexist? Asian American Journal of Psychology, 4(1), 30-40.

Comissão Econômica para a América Latina e o Caribe - CEPAL. (2015). Estudio Económico de América Latina y el Caribe 2015: desafios para impulsar el ciclo de inversión con miras a reactivar el crecimiento. Santiago: CEPAL. Recuperado el 05 de octubre de 2019, de https://repositorio.cepal.org/bitstream/handle/11362/38713/S1500733_es.pdf?sequence=112\&isAll owed $=y$

Chile. Departamento de Extranjería y Migración. Estadísticas Migratorias, Registros Administrativos del Departamento de Extrajería y Migración. (2018). Recuperado de https:/www.extranjeria.gob.cl/estadisticas-migratorias/.

Dudek-Shriber, L. (2004). Parent stress in the neonatal intensive care unit and the influence of parent and infant characteristics. American Journal of Occupational Therapy, 58(5), 509-520. Recuperado el 05 de noviembre de 2019, de https://eds-b-

ebscohostcom.ezp.lib.unimelb.edu.au/eds/detail/detail?vid=0\&sid=c774fb0f-1de8-4d52-9dffeac923134508\%40pdc-v-sessmgr01\&bdata=JnNpdGU9ZWRzLWxpdmU\%3d

Fair, F., Raben, L., Watson, H., Vivilaki, V., Muijsenbergh, M., Soltani, H., \& ORAMMA team. (2020). Migrant women's experiences of pregnancy, childbirth and maternity care in European countries: a systematic review. PLoS One, 15(2), 1-26. http://dx.doi.org/10.1371/journal.pone.0228378.

Fredriksen, E., Soest, T., Smith, L., \& Moe, V. (2019). Parenting stress plays a mediating role in the prediction of early child development from both parents' perinatal depressive symptoms. Journal of Abnormal Child Psychology, 47(1), 149-164. http://dx.doi.org/10.1007/s10802-018-0428-4.

García, S., Duarte, L., \& Mejías, M. D. C. (2014). Afrontamiento de la familia ante el nacimiento de un hijo prematuro. NURE Investigación, (69),02-11. Recuperado el 15 de noviembre de 2019, de http://dialnet.unirioja.es/servlet/articulo? $\operatorname{codigo}=4740160 \&$ info=resumen $\&$ idioma $=\mathrm{ENG}$

García-García, J., Pardo-Serrano, C., Hernández-Martínez, A., Lorenzo-Díaz, M., \& Gil-González, D. (2008). Diferencias obstétricas y neonatales entre mujeres autóctonas e inmigrantes. Progresos de Obstetricia y Ginecologia, 51(2), 53-62.

Gjerdingen, D., McGovern, P., Attanasio, L., Johnson, P. J., \& Kozhimannil, K. B. (2014). Maternal depressive symptoms, employment, and social support. Journal of the American Board of Family Medicine, 27(1), 87-96. http://dx.doi.org/10.3122/jabfm.2014.01.130126.

Harding, C., Levin, A., Crossley, S. L., Murphy, R., \& van den Engel-Hoek, L. (2019). Effects of early communication intervention on speech and communication skills of preterm infants in the neonatal intensive care unit (NICU): a systematic review. Journal of Neonatal Nursing, 25(4), 177-188. http://dx.doi.org/10.1016/j.jnn.2019.04.004.

Higginbottom, G. M. A., Hadziabdic, E., Yohani, S., \& Paton, P. (2014). Immigrant women's experience of maternity services in Canada: a meta-ethnography. Midwifery, 30(5), 544-559. 
Ho, H. Z., Chen, W. W., Tran, C. N., \& Ko, C. T. (2010). Parental involvement in Taiwanese families: father-mother differences. Childhood Education, 86(6), 376-381.

http://dx.doi.org/10.1080/00094056.2010.10523173.

Ionio, C., Lista, G., Mascheroni, E., Olivari, M. G., Confalonieri, E., Mastrangelo, M., \& Scelsa, B. (2017). Premature birth: complexities and difficulties in building the mother-child relationship. Journal of Reproductive and Infant Psychology, 35(5), 509-523.

Jeong, H. G., Lim, J. S., Lee, M. S., Kim, S. H., Jung, I. K., \& Joe, S. H. (2013). The association of psychosocial factors and obstetric history with depression in pregnant women: focus on the role of emotional support. General hospital psychiatry, 35(4), 354-358.

Jiménez, F., Loscertales, M., Martínez, A., Barbancho, M. M., Lanzarote, M. D., Macías, C., \& Nieto, C. (2003). Padres de recién nacidos ingresados en UCIN, impacto emocional y familiar. Vox Paediatrica, 11(2), 27-33. Recuperado el 22 de noviembre de 2019, de https://idus.us.es/bitstream/handle/11441/54413/Voxpaed11.2pags2733.pdf? sequence $=1 \&$ isAllowed $=y$

Kartzow, R. V., \& Castillo-Durán, C. (2012). Embarazo y parto en madres inmigrantes en Santiago, Chile. Revista Chilena de Pediatria, 83(4), 366-370. http://dx.doi.org/10.4067/S037041062012000400007.

Kasat, K., Stoffels, G., \& Ellington, M. (2020). Improving communication with parents: the Neonatal Intensive Care Unit Empathy Workshop. Journal of Perinatology, 40(9), 1423-1432. http://dx.doi.org/10.1038/s41372-020-0742-x.

Kiang, L., Glatz, T., \& Buchanan, C. M. (2017). Acculturation conflict, cultural parenting self-efficacy, and perceived parenting competence in Asian American and Latino/a families. Family Process, 56(4), 943-961. http://dx.doi.org/10.1111/famp.12266.

Koroleff, P. T. (2001). La creación progresiva del vínculo madre-niño. Revista de Psicología, 19(2), 305318.

Liu, T. T., Lei, M. J., Li, Y. F., Liu, Y. Q., Meng, L. N., \& Jin, C. D. (2018). Effects of parental involvement in infant care in neonatal intensive care units: a meta-analysis. Frontiers of Nursing, 5(3), 207-215. http://dx.doi.org/10.1515/fon-2018-0028.

Lotterman, J. H., Lorenz, J. M., \& Bonanno, G. A. (2019). You can't take your baby home yet: a longitudinal study of psychological symptoms in mothers of infants hospitalized in the NICU. Journal of Clinical Psychology in Medical Settings, 26(1), 116-122. http://dx.doi.org/10.1007/s10880018-9570-y.

Martínez, F. T. (2012). Fenomenología como método de investigación: Una opción para el profesional de enfermería. Revista de Enfermería Neurológica, 11(2), 98-101. Recuperado el 20 de octubre de 2019, de https://www.medigraphic.com/pdfs/enfneu/ene-2012/ene122h.pdf

Miller, L. S., Robinson, J. A., \& Cibula, D. A. (2016). Healthy immigrant effect: preterm births among immigrants and refugees in Syracuse, NY. Maternal and child health Journal, 20(2), 484-493.

Molina, M. E. (2006). Transformaciones histórico culturales del concepto de maternidad y sus repercusiones en la identidad de la mujer. Psykhe, 15(2), 93-103. http://dx.doi.org/10.4067/S071822282006000200009.

Nix, C. M., \& Ansermet, F. (2009). Prematurity, risk factors, and protective factors. In C. H. Zeanah Jr (Ed.), Handbook of infant mental health (pp. 180-196). New York: Guilford Pres.

Ojeda, M., Rodriguez, L., Rodríguez, J., Rauld, J., \& Cofré, G. (2018). Características de la inmigración internacional en Chile, Censo 2017. Santiago: INE. Recuperado el 16 de octubre de 2019, de http://www.censo2017.cl/descargas/inmigracion/181123-documento-migracion.pdf

Organización Integral para las Migraciones - OIM. (2018). Informe sobre las migraciones en el mundo 2018. Genebra: OIM.

Otzen, T., \& Manterola, C. (2017). Técnicas de muestreo sobre una población a estudio. International Journal of Morphology, 35(1), 227-232. http://dx.doi.org/10.4067/S0717-95022017000100037. 
Parra, E., Sánchez-Gómez, M. C., García-Peñalvo, F. J., Sánchez, J. L. M., Martín-Cilleros, M. V., Franco, M., \& Bruno, A. P. (2017). Análisis de contenido cualitativo: estudio de la satisfacción de los usuarios sobre la presentación de un nuevo medicamento en la salud pública. In A. P. Costa, M. C. Sánchez-Gómez \& M. V. Martín-Cilleros (Eds.), La Práctica de la investigación cualitativa: Ejemplificación de estudios (pp. 57-92). São Paulo: Ludomedia.

Pham, T., Hua, A., Li, D., Bar, S., Spinazzola, R., \& Milanaik, R. (2018). Leading stress factors reported by parents of Nicu infants. Pediatrics, 141(1), 533. http://dx.doi.org/10.1542/peds.141.1_meetingabstract.533.

Puthussery, S. (2016). Perinatal outcomes among migrant mothers in the United Kingdom: is it a matter of biology, behaviour, policy, social determinants or access to health care? Best Practice \& Research. Clinical Obstetrics \& Gynaecology, 32, 39-49. http://dx.doi.org/10.1016/j.bpobgyn.2015.09.003.

Ramos-Vidal, I. (2011). Identificando áreas sensibles en los contactos interculturales: un estudio exploratorio. Revista de Psicología, 29(1), 67-99.

Roller, M. R. (2019). A quality approach to qualitative content analysis: similarities and differences compared to other qualitative methods. Forum Qualitative Social Research, 20(3), 1-21.

Rossel, C. K., Carreño, T., \& Maldonado, M. E. (2002). Afectividad en madres de niños prematuros hospitalizados. Un mundo desconocido. Revista Chilena de Pediatria, 73(1), 15-21. http://dx.doi.org/10.4067/S0370-41062002000100004.

Royo, R. (2011). Maternidad, paternidad y conciliación en la CAE: ¿Es el trabajo familiar un trabajo de mujeres? Bilbao: Universidad de Deusto. Recuperado el 20 de octubre de 2019, de http://www.deusto-publicaciones.es/deusto/pdfs/otraspub/otraspub02.pdf

Sánchez-Fernández, Y., Muñoz-Ávalos, N., Pérez-Cuadrado, S., Robledo-Sánchez, A., Pallás-Alonso, C. R., Cruz-Bértolo, J., \& García, A. (2003). Mujeres inmigrantes: características del control prenatal y del parto. España: Elsevier.

Savage, C. L., Anthony, J., Lee, R., Kappesser, M. L., \& Rose, B. (2007). The culture of pregnancy and infant care in African American women: an ethnographic study. Journal of Transcultural Nursing, 18(3), 215-223. http://dx.doi.org/10.1177/1043659607301294.

Shaw, R. J., Deblois, T., Ikuta, L., Ginzburg, K., Fleisher, B., \& Koopman, C. (2006). Acute stress disorder among parents of infants in the neonatal intensive care nursery. Psychosomatics, 47(3), 206212. http://dx.doi.org/10.1176/appi.psy.47.3.206.

Smith, M. D., \& Floro, M. S. (2020). Food insecurity, gender, and international migration in low-and middle-income countries. Food Policy, 91, 1-60. http://dx.doi.org/10.1016/j.foodpol.2020.101837.

Stefoni, C. (2017). Panorama de la migración internacional en América del Sur. Santiago: CEPAL. Recuperado el 01 de noviembre de 2019, de https://refugeesmigrants.un.org/sites/default/files/eclac_america_del_sur_resumen_ejecutivo.pdf

Stern, D. N. (2004). The motherhood constellation: therapeutic approaches to early relational problems. In A. J. Sameroff, S. C. McDonough \& K. L. Rosenblum (Eds.), Treating parent-infant relationship problems: Strategies for intervention (pp. 29-42). New York: Guilford Press.

Telzer, E. H. (2010). Expanding the acculturation gap-distress model: an integrative review of research. Human Development, 53, 313-340. http://dx.doi.org/10.1159/000322476.

Trumello, C., Candelori, C., Cofini, M., Cimino, S., Cerniglia, L., Paciello, M., \& Babore, A. (2018). Mothers' depression, anxiety, and mental representations after preterm birth: a study during the infant's hospitalization in a Neonatal Intensive Care Unit. Frontiers in Public Health, 6, 2-9. http://dx.doi.org/10.3389/fpubh.2018.00359.

Turner, M., Chur-Hansen, A., Winefield, H., \& Stanners, M. (2015). The assessment of parental stress and support in the neonatal intensive care unit using the Parent Stress Scale - Neonatal Intensive Care Unit. Women and Birth; Journal of the Australian College of Midwives, 28(3), 252-258. http://dx.doi.org/10.1016/j.wombi.2015.04.001.

Wikberg, A. M. (2020). A theory on intercultural caring in maternity care. Scandinavian Journal of Caring Sciences, 35(2), 442-456. http://dx.doi.org/10.1111/scs.12856. 


\section{Contribución de los Autores}

Andrea Mira es el responsable de coordinación del equipo de investigación, construcción del estudio, realización y codificación de las entrevistas, análisis de los resultados, redacción y edición del texto. Rodolfo Bastías es el responsable de construcción del estudio, coordinación del trabajo de campo, realización y colaboración en la redacción del texto. Todos los autores aproban la version final del texto.

\section{Autor para la correspondencia}

Andrea Mira

e-mail: andrea.mira@unab.cl

\section{Editora de sección}

Profa. Dra. Luzia Iara Pfeifer 